\title{
Model for Profiler Agent during unexpected educational circumstances
}

\section{Georgi Cholakov, Asya Stoyanova-Doycheva}

Department of Computer Systems, University of Plovdiv "Paisii Hilendarski", Bulgaria.

\begin{abstract}
Nowadays distance education helps when no other traditional possibility is allowed. But is it a good alternative to replace entirely the traditional education? Could it provide at least the same level of quality or it brings problems that we are not prepared for? The paper presents an observation of a problem with keeping students focused on their education - constant dropping of engagement and unintentional loss of attention during situation of distance learning, and a practical approach to deal with the problem. Appling measures to keep students' attraction led to the need of some aspects' automation - a model for a new intelligent assistant, software agent, was developed, along with the current ones in the existing system. This assistant will create profiles of students, helping with personalized tracking of each student's progress in specific subject, recommending topics to improve knowledge and fill knowledge gaps. It will "live" in the extension of an existing system and cooperate with other agents to accomplish its goals, proactively assisting in students' learning aspects, as well as teachers' efforts to prepare better and more suitable educational materials.
\end{abstract}

Keywords: E-learning; software agents; service-oriented architectures; educational portal; personal assistants. 


\section{Introduction}

In the last year people have faced situation, that most of them were unfamiliar with - social and physical distance, isolation. We had to switch from a "normal" life to some kind of strange being, making us rethink what are the most important priorities and how we could live, communicate and interact using only technologies, without close personal and physical contact.

As this situation spread over entire world and almost any industry, it has not skipped the education, as well. Everyone involved in educational processes had to learn how to deal with the systems for distance learning - teachers faced the need to prepare their teaching materials and attitude for distance learning and e-learning. It is obvious that e-learning itself is something very useful, but now it seems that it can't replace traditional learning entirely, or at least not without undesired side effects. And probably among the most challenging problems was how to keep students attracted to the matter and education as a whole; they had to learn with fast pace how to educate themselves when no one is around physically to help them (which was one of the positive effects, building problem solving skills for many), but meantime they need to keep their focus on education, not being distracted by the laziness and the comfort of addiction of using their smart devices only for fun. The last has become the main challenge according to our experience, because the students' engagement is continuously dropping as our observations show below. As a result, we had to respond by taking adequate measures, and one of them is the subject of this article.

It turns out that the problem described above is not isolated. Many institutions experience demotivation of students, distraction and lack of attention - and respectively, how the manage it. Their studies on the problem are described in (Hornsby, 2020; Reynolds, et al., 2020).

Many systems helped teachers and students keep in touch in education - like Google Classroom (Google, 2014), Moodle (Dougiamas, 2002), to name a few. Many universities have developed their own systems to cover their specific needs, among them is Plovdiv University "Paisii Hilendarski" (Plovdiv, Bulgaria), where we have implemented and extended Liferay (Liferay Inc., 2000) based portal named DeLC (Doychev, 2013), with several satellite extensions, subjects of many studies (Stoyanov, et al., 2012; Stoyanov, et al., 2005).

Distributed eLearning Center (DeLC) is intended to support the educational process for both parties involved - students and teachers, and it has proven its capabilities for more than ten years of use. For students, it provides better education quality and knowledge improvements, filling gaps in understanding the course materials; for teachers - improvement of their lectures and teaching methods, and mostly in students' examination. Many aspects concerning DeLC's work are constantly monitored and this portal is periodically a subject of improvements, which is true for the teaching materials as well. 
During its lifecycle, the architecture was extended with different subsystems, among which is IntelliDeLC - to provide a personalized e-learning environment with reactive and proactive behavior (Stoyanov, et al., 2011; Cholakov, 2013; Stoyanov, et al., 2012). The proactivity improves the usability and friendliness of the system to the users, which means that the software can operate ,on behalf of the user” and ,activate itself” when it ,estimates” that its intervention is needed. In DeLC, proactivity is ensured through ,reinforcement” of the service-oriented architecture with intelligent components - which are software agents. These agents (also called assistants) "live" and operate in the back-end agent-oriented server.

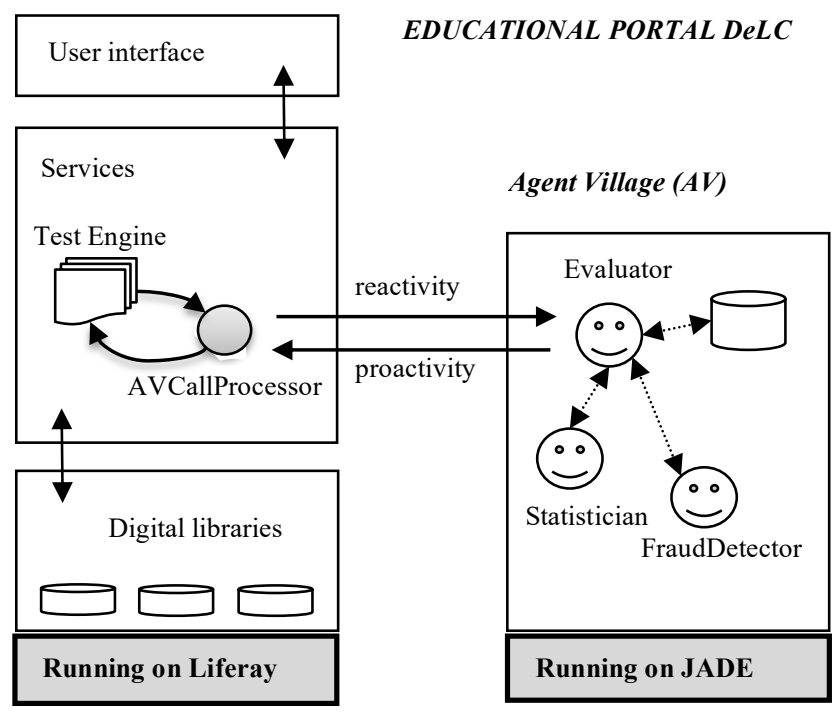

Figure 1. DeLC architecture with Agent Village extension.

Their environment is referred to as Agent Village (AV). The DeLC architecture is simply depicted on figure 1 .

\subsection{Agent Village}

The Agent Village is the environment where the assistants (agents) are deployed and operate. It is created and running on JADE framework (Telecom Italia, n.d.). The communication between agents is based on ACL messages (FIPA, 2021). Outside Agent Village, the agents' services are wrapped and available as SOAP web services. More details on this topic are published in (Cholakov, 2013). 


\subsection{AVCallProcessor}

This module serves as a mediator during the communication between DeLC and Agent Village. It is deployed in the portal as a system service and makes all requests to agents transparent for the portal. The communication works as follows: when DeLC needs a service from the Agent Village it simply calls a method from AVCallProcessor, which generates a SOAP request to Agent Village, where this request is transformed to an ACL message, understandable by the agents; on the way back, the result is formed by the corresponding agent as an ACL message, which is transformed by Agent Village as a SOAP response, transferred to AVCallProcessor, which parses the response and generates the result in expected format by the portal.

\subsection{Evaluator Agent}

The Evaluator Agent (EA) provides expert assistance to the teacher in the assessment of electronic tests. While the test engine in DeLC has a system service for automated assessment of "multiple choice questions", this assistant helps in analyzing answers in short free-text questions by rating the answer, but always leaving the last word to the teacher. When the test engine needs external assessment, it initiates a request for expert assistance, and then the reactive behavior of the EA is applied. The agent uses knowledge base to search for matches, generated from keywords and phrases for each test question. Latest approbation of this agent was published in (Cholakov, 2020).

\subsection{FraudDetector}

The FraudDetector agent helps trying to recognize any attempts for cheating in the answers, given by the students. Among these attempts are copy/paste results from Internet search engines and using portal's chat system to share answers. This assistant cooperates with Evaluator and if its receptors detect a probability of a cheating, it informs Evaluator, which, on the other hand, informs the assessing teacher that this answer requires a special attention, because it is a suspicious one. This agent relies on its own knowledge base, which is generated from: the words that forms each question; the keywords for each question; the words from the students' answers; the messages between students in the portal's chat system, which are marked by an operator as cheating. More on this in (Cholakov, 2013).

\subsection{Statistician}

Statistician stores information about all processed answers with full history of the details from all calculating methods, used by the Evaluator agent. This assistant needs a feedback how many points are finally given by the teacher for each answer. Thus, it accumulates a knowledge base for each teacher and can decide which of the methods best suits the assessment style of the currently assessing teacher. 


\section{The problem}

During the pandemic measures we had to switch to distance learning entirely. Prolonging this period showed that the students' interest to educational process is constantly dropping figure 2 depicts this statistic from three semesters.

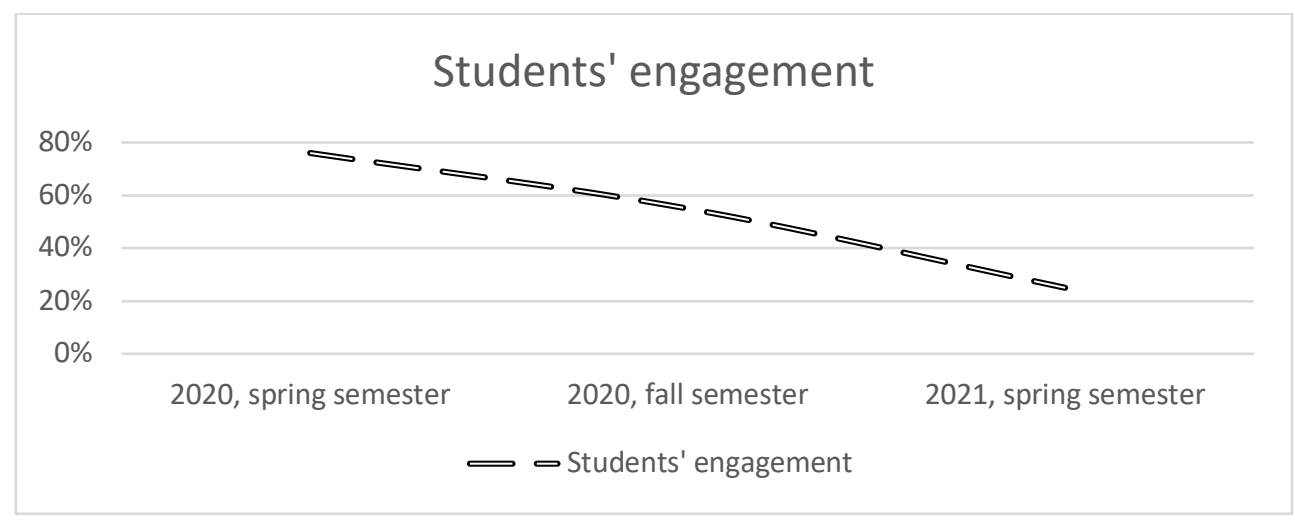

Figure 2. Students' interest during last three semesters.

The statistics shown above take into account an average students' attendance, classwork, as well as taking part actively during lections and labs in a particular subject - "Database management systems", in four different specialties, but the situation is similar to these ones in other subjects. So, we needed to react in a timely manner not to lose them along the way. As part of our weekly tasks, we started putting students on tests and provoking quizzes after each new topic, more often than usually. As a result, now students have more tasks, but these tasks do not consider individual progress and gaps, because they are class based. Controlling each student individually turned out to be a heavy process, as we have an average of over two hundred students per semester per subject, which means that we need some automation to help keeping the quality of education.

\section{The idea}

Since we have automated the process of exams evaluation, which is individualized per student, can we have something similar to personalize the quizzes and weekly tests, based on previous results? And end up monitoring students progress, as others do (Dongming, et al., 2014; Costa, et al., 2019).

Apparently, we need software assistant, that could create personal profile for each student per subject, which should be capable to recognize knowledge gaps and choose appropriate materials and quizzes for each. The assistant's model, called Profiler, that emerged from the business analysis, shows next necessary features: 
- Tracking progress for each student per subject - this information could be extracted from the tests results, available in portal's database - and thus profiling each student. Using data mining could reveal patterns of behavior while learning, not obvious for teachers, but useful for analysis and further directions - which implies active cooperation with Evaluator agent, which has knowledge about past student's results;

- Based on the profile above, the assistant should create personalized tests with content that seems to be not very well learned by the student - this should keep student on track. It could recommend materials - lectures, books' chapters, depending on individual progress, and that requires each topic to have related educational resources. This functionality implies using of AI methods to determine the level of knowledge perception;

- To honor student's efforts, the assistant could take part in final evaluation of the grade, because it will keep information in the profile about student's results during the entire semester;

- Analyzing the average progress for each topic of the materials, this assistant should give a feedback to teacher as well, to propose materials updates when necessary.

We consider this functionality as a good base to start implementation, although there are still some points to analyze. The architecture of Agent Village will be extended with the new agent as shown on figure 3 .

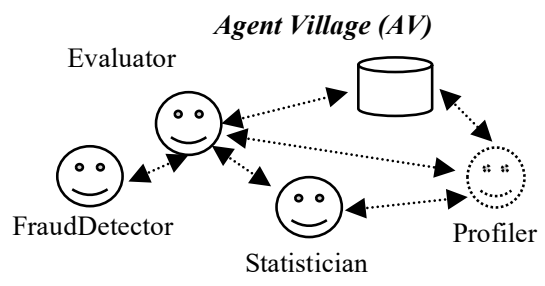

Figure 3. AV extended architecture.

Getting deeper into business analysis of the topic revealed that the problem has psychological aspects - beyond the scope of a simple development of single component (Profiler agent), and they should be taken into account in requirement specification. That's why using Agile methodology for this development seems a natural choice - dividing the task into several small iterations (sprints) will help in getting first impressions faster, and give more time to receive opinions from specialists in human behavior. Such approach would produce more reliable results after each iteration and would help in producing more useful software component, eventually.

\section{Conclusion}

Keeping high education quality level during distance learning is crucial for both parties involved - students and teachers. Applying new measures to engage students with the 
educational process puts new challenges. To automate some frequent tasks, we need to use machine assistance - in our case to develop new software agent, responsible for profiling and control of students about their progress in particular subject. This assistant should help students get better knowledge and it could bring even more value - generating psychological effect that the education goes on, it is not suspended, and students must give their best in this process. The expectations are this agent to help proactively students in learning, as well as the teachers in preparing lectures content, and provide monitoring over students' progress. Now, as we have the requirements, the development process could start.

\section{Acknowledgements}

The research is partly supported by the project FP21-FMI-002 "Intelligent innovative ICT in research in mathematics, informatics and pedagogy in education" of the Scientific Fund of the University of Plovdiv "Paisii Hilendarski".

\section{References}

Cholakov, G. (2013). Hybrid Architecture for Building Distributed Center for e-Learning. PhD Thesis. Plovdiv, Bulgaria.

Cholakov, G. (2020). Approbation of software agent Evaluator in a nonspecific environment for extension of its purpose. 2020 International Conference Automatics and Informatics (ICAI), (pp. pp. 1-5). Varna, Bulgaria. doi:10.1109/ICAI50593.2020.9311346

Costa, L., Souza, M., Salvador, L., \& Amorim, R. (2019). Monitoring Students Performance in E-Learning Based on Learning Analytics and Learning Educational Objectives. 19th International Conference on Advanced Learning Technologies (ICALT) (pp. pp. 192193). Maceió, Brazil: IEEE.

Dongming, X., Huang, W., Wang, H., \& Jon, H. (2014). Enhancing e-learning effectiveness using an intelligent agent-supported personalized virtual learning environment: An empirical investigation. Information \& Management (Elsevier)(51(4)), pp. 430-440.

Dougiamas, M. (2002, August). Retrieved from Moodle: https://moodle.org/

Doychev, E. (2013). Environment for Provision of eLearning Services. PhD Thesis. Plovdiv, Bulgaria.

FIPA. (2021, February). Agent Communication Language Specifications. Retrieved from http://www.fipa.org/repository/aclspecs.html

Google. (2014, August). (Google) Retrieved February 2021, from Google Classroom: https://classroom.google.com

Hornsby, D. (2020). Moving large classes online: Principles for teaching, learning and assessment. Pedagogy for Higher Education Large Classes (PHELC20) Co-located with 6th International Conference on Higher Education Advances (HEAd'20). Valencia, Spain. doi:10.5281/zenodo.3893426

Liferay Inc. (2000). Liferay. Retrieved from https://www.liferay.com/ 
Reynolds, J., Cai, V., Choi, J., Faller, S., Hu, M., Kozhumam, A., . . Vohra, A. (2020). Teaching during a pandemic: Using high-impact writing assignments to balance rigor, engagement, flexibility, and workload. Ecology and Evolution, 10(22). doi:10.1002/ece3.6776

Stoyanov, S., Doychev, E., Valkanova, V., \& Cholakov, G. (2012). Education Cluster for Intelligent Provision of eLearning Services. DBKDA 2012: The Fourth International Conference on Advances in Databases, Knowledge, and Data Applications, Reunion, IARIA, (pp. pp. 45-50).

Stoyanov, S., Ganchev, I., O’Droma, M., Doychev, E., \& Cholakov, G. (2005). Agentoriented Distributed eLearning Center. Proceedings of the 2th Balkan Conference in Informatics BCI2005, (pp. pp. 238-245). Ohrid, Macedonia.

Stoyanov, S., Valkanova, V., Cholakov, G., \& Sandalski, M. (2011). Education Portal for Reactive and Proactive Service Provision. COGNITIVE 2011: The Third International Conference on Advanced Cognitive Technologies and Applications, (pp. pp. 99-103). Rome.

Stoyanov, S., Zedan, H., Doychev, E., Valkanov, V., Popchev, I., Cholakov, G., \& Sandalski, M. (2012). Intelligent Distributed eLearning Architecture. In V. M. Koleshko (Ed.), Intelligent Systems (pp. pp. 185-218). InTech.

Telecom Italia. (n.d.). JADE. Retrieved February 2021, from https://jade.tilab.com/ 